\title{
Behavioral Modulation of Tactile Responses in the Rat Somatosensory System
}

\author{
Erika E. Fanselow and Miguel A. L. Nicolelis \\ Department of Neurobiology, Duke University Medical Center, Durham, North Carolina 27710
}

We investigated the influence of four different behavioral states on tactile responses recorded simultaneously via arrays of microwires chronically implanted in the vibrissal representations of the rat ventral posterior medial nucleus (VPM) of the thalamus and the primary somatosensory cortex (SI). Brief (100 $\mu \mathrm{sec})$ electrical stimuli delivered via a cuff electrode to the infraorbital nerve yielded robust sensory responses in VPM and SI during states of quiet immobility. However, significant reductions in tactile response magnitude and latency were observed in VPM and SI during large-amplitude, exploratory movements of the whiskers (at $\sim 4-6 \mathrm{~Hz}$ ). During small-amplitude, 7-12 Hz whisker-twitching movements, a significant reduction in SI response magnitude and an increase in VPM and SI response latencies were observed as well. When pairs of stimuli with interstimulus intervals $<100 \mathrm{msec}$ were delivered during quiet immobility, the response to the second stimulus in the pair was reduced and occurred at a longer latency compared with the response to the first stimulus. In contrast, during large- amplitude whisker movements and general motor activity, paired stimuli yielded similar sensory responses at interstimulus intervals $>25 \mathrm{msec}$. These response patterns were correlated with the amount and duration of postexcitatory firing suppression observed in VPM and SI during each of these behaviors. On the basis of these results, we propose that sensory responses are dynamically modulated during active tactile exploration to optimize detection of different types of stimuli. During quiet immobility, the somatosensory system seems to be optimally tuned to detect the presence of single stimuli. In contrast, during whisker movements and other exploratory behaviors, the system is primed to detect the occurrence of rapid sequences of tactile stimuli, which are likely to be generated by multiple whisker contacts with objects during exploratory activity.

Key words: gating; postexcitatory inhibition; somatosensory; sensory motor integration; behavioral context; $\mu$ oscillations; whisking
As animals actively explore their environment, tactile stimuli are not experienced as isolated, individual events but, instead, are sampled along with multiple contextual factors, as well as other stimuli. If one is to understand how sensory stimuli are processed, it is vital to take into account the fact that sensory response properties are dynamic (Chapin and Woodward, 1981, 1982a,b; Nelson, 1984; Nicolelis and Chapin, 1994; Castro-Alamancos and Connors, 1996a,b; Ghazanfar and Nicolelis, 1997) and that contextual factors, such as the type of movement used to explore the environment and the spatiotemporal nature of the stimulus, may affect the basic characteristics of sensory responses. The rationale for this study derives from experimental evidence that motor activity alters the characteristics of neural responses to tactile stimulation. For instance, forelimb movements have been shown to alter responses to somatosensory stimuli in the rat primary somatosensory cortex (SI) (Chapin and Woodward, 1981, 1982a,b; Shin and Chapin, 1990b), the rat ventral posterior lateral thalamus (Shin and Chapin, 1990a,b), rat dorsal column nuclei (O'Keefe and Gaffan, 1971), the medial lemniscus pathway in cat (Ghez and Lenzi, 1971; Coulter, 1974), and in the monkey medial lemniscus, somatosensory thalamus, and somatosensory cortex

\footnotetext{
Received Dec. 11, 1998; revised June 7, 1999; accepted June 10, 1999.

This work was supported by the Klingenstein Foundation, the Whitehall Foundation, the National Institute of Dental Research Grant DE-11121-01 to M.A.L.N., and a National Science Foundation predoctoral fellowship to E.E.F. We would like to thank Merri Rosen for her assistance in preliminary data collection and Dr. Mike Weliky for assisting with cuff electrode design.

Correspondence should be addressed to Erika E. Fanselow, Department of Neurobiology, Box 3209, Duke University Medical Center, Durham, NC 27710. Copyright (C) 1999 Society for Neuroscience $0270-6474 / 99 / 197603-14 \$ 05.00 / 0$
}

(Chapman et al., 1988). In addition, cortical responses to vibrotactile stimulation of the hand have been shown to undergo several types of modifications when motor activity is anticipated (Nelson, 1984; Lebedev et al., 1994). These studies point to the existence of mechanisms by which the nervous system can modulate tactile responses during the execution of exploratory movements.

As rats gather tactile information about their environment, they actively move their whiskers across objects or surfaces in repeated rhythmic sweeps (Welker, 1964; Carvell and Simons, 1990). Under these conditions, the trigeminal somatosensory system is subjected to a barrage of ascending neuronal activity resulting from multiple mechanical contacts of the whiskers with the target object. It is clear that neural responses in the whisker representations of the rat ventral posterior medial nucleus of the thalamus (VPM) and the SI cortex can integrate stimuli from multiple vibrissae across poststimulus time (Simons, 1985; Simons and Carvell, 1989; Ghazanfar and Nicolelis, 1997). However, it remains unknown how this integration occurs during different behavioral situations in which distinct types of exploratory movements are used by the animal. This study addressed this issue by investigating how tactile stimuli are integrated during four different behaviors that can be easily identified in freely moving rats. These behaviors included quiet immobility; largeamplitude, low-frequency $(4-6 \mathrm{~Hz})$ exploratory-whisking movements; small-amplitude, high-frequency (7-12 Hz) whiskertwitching movements accompanied by $7-12 \mathrm{~Hz}$ neural oscillations in VPM and SI; and general motor activity without concurrent whisker movements. 
In this paper, the potential modulatory effects of the four different behavioral states described above were characterized by simultaneously recording the extracellular activity of populations of neurons located in the vibrissal representations of VPM and SI. Special emphasis was put on investigating tactile response modulation during different types of whisker movements. Reproducible patterns of tactile stimuli were obtained by delivering brief electrical pulses to the infraorbital branch of the trigeminal nerve using a chronically implanted nerve cuff electrode. We observed that different exploratory movements induced modulation of sensory responses in both VPM thalamus and SI cortex, suggesting that the somatosensory system actively modulates tactile input to optimize the detection of specific types of stimulation under different behavioral conditions.

\section{MATERIALS AND METHODS}

Cuff electrode construction. Nerve cuff electrodes were used to deliver uniform electrical stimulation to the infraorbital nerve, the branch of the sensory division of the trigeminal nerve that carries tactile information from the facial vibrissae to the trigeminal ganglion. These cuff electrodes were designed to fit around the circumference of the infraorbital nerve and were custom made (Fig. 1a). The cuffs contained two bands of platinum (Goodfellow, Berwyn, PA) $0.5 \mathrm{~mm}$ wide and $0.025 \mathrm{~mm}$ thick. Each band was connected to a piece of flexible, three-stranded, Tefloncoated wire (AM Systems, Everett, WA) that was used to pass current between the bands. The bands were located $\sim 0.8 \mathrm{~mm}$ apart from one another within the cuff. The platinum bands and places of attachment to the lead wires were embedded in a thin film of Sylgard (Factor II, Lakeside, AZ) that served as a substrate to hold the electrode together as well as to insulate the platinum electrically from the surrounding tissue. The face of each band that contacted the nerve inside the cuff was left exposed. A piece of surgical silk was attached to the outside of the Sylgard for use in maneuvering the electrode during implantation. The inner diameter of the finished cuff was $\sim 1.7 \mathrm{~mm}$.

Subjects. Outbred, female Long-Evans hooded rats weighing 280-350 gm served as the subjects in this study. The results reported here were observed in a total of eight rats. Five of these with the most complete data sets were used for the statistical analyses presented in this report.

Procedures for chronic implantation of nerve cuff electrodes and microwire electrodes. Rats received chronic implants of microwires in both the VPM thalamus and the SI cortex and a chronic cuff electrode implant around the infraorbital nerve under sodium pentobarbital anesthesia (50 $\mathrm{mg} / \mathrm{kg}$, i.p.). Anesthesia was maintained throughout the surgery such that animals were not responsive to foot pinch, breathing was slow and regular, and the blinking reflex was absent. Supplemental doses of pentobarbital $(0.05 \mathrm{cc})$ were administered as needed. During the surgery, four to five stainless steel screws were screwed into the skull to serve as electrical grounds and as anchors for cementing the microwires in place after implantation.

Two small craniotomies were performed, one above the whisker representation of the SI cortex and another located above the VPM thalamus. Coordinates for the craniotomies (VPM, rostrocaudal, $-3.0 \mathrm{~mm}$; mediolateral, $3.0 \mathrm{~mm} ; 5.0 \mathrm{~mm}$ down; SI, rostrocaudal, $-3.0 \mathrm{~mm}$; mediolateral, $5.5 \mathrm{~mm} ; 1.3 \mathrm{~mm}$ down; measurements relative to bregma) were obtained from a rat brain atlas by Paxinos and Watson (1986). Prefabricated stainless steel microwire arrays and bundles containing 16 wires apiece (NBLabs, Dennison, TX) were implanted into these target areas. First, a bundle of 16 microwires was slowly lowered into the VPM thalamus. Wires in the bundles were trimmed at an angle such that the microwires could sample a range of barreloids in the VPM nucleus. Recordings of neural activity and receptive field assessments were performed while lowering the electrodes to position them correctly. When the correct coordinates for VPM were reached and robust responses to whisker stimulation were observed, the bundle was cemented with dental acrylic to the skull and to screws drilled into the skull. Subsequently, the dura above the SI whisker representation was resected, and an array of 16 blunt-tipped $50 \mu \mathrm{m}$ microwires (measurement includes Teflon coating) was implanted into layer V of the SI whisker representation. Wires within the arrays were aligned in two rows of eight wires, with each row spaced $0.5 \mathrm{~mm}$ apart and electrodes spaced $200 \mu \mathrm{m}$ from one another within the rows. Wires were held in this configuration using epoxy and a polyethylene glycol coating applied during electrode manufacture and were a
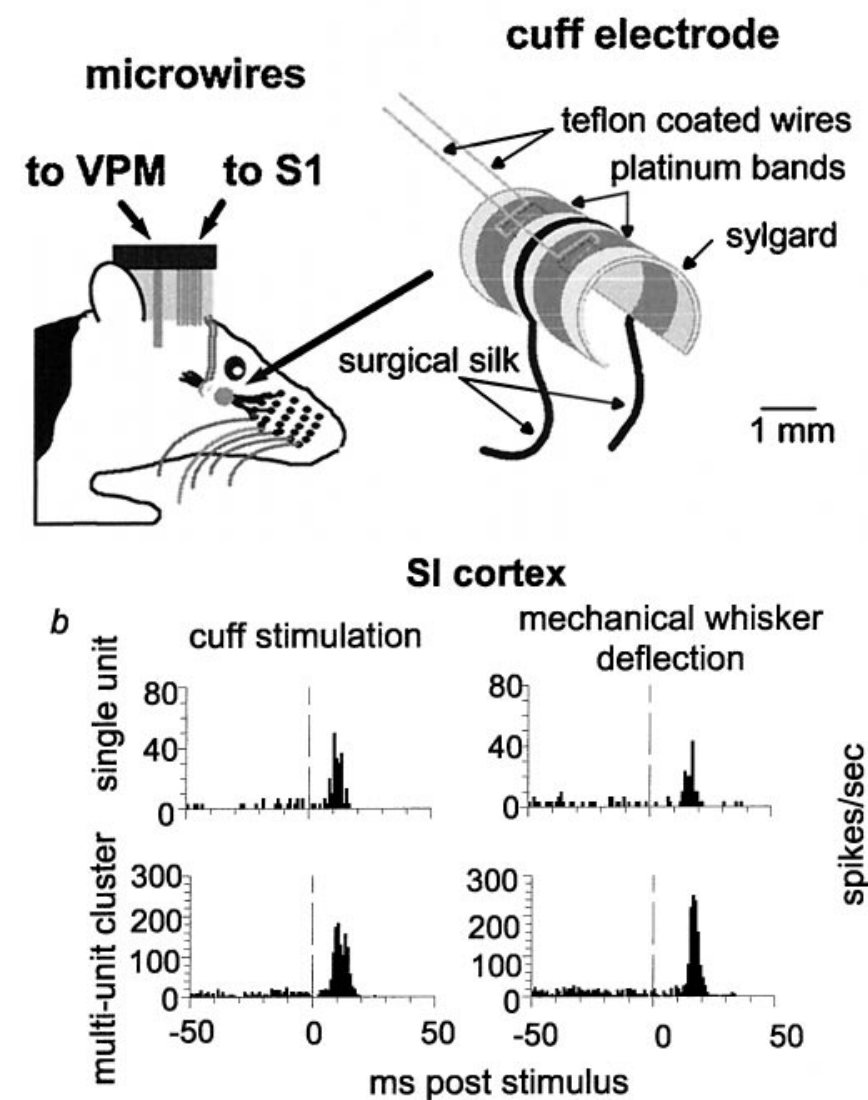

$C$

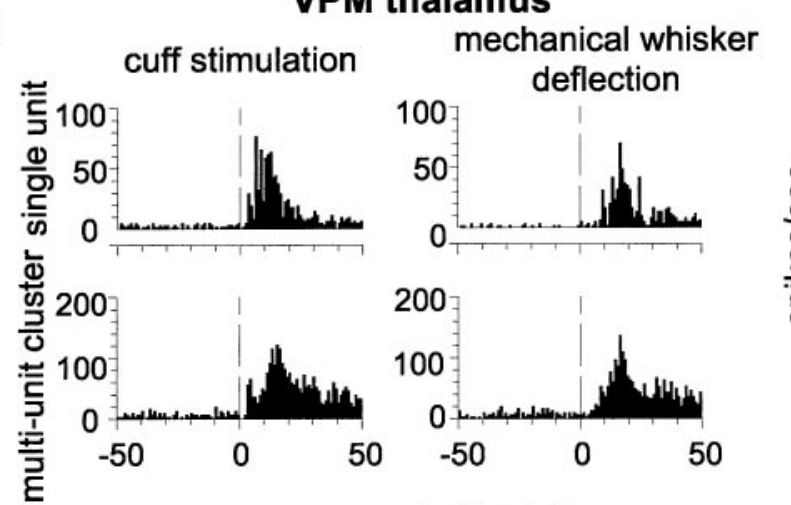

क्ष
के
के

Figure 1. Nerve cuff electrode design and comparison of sensory responses in VPM and SI resulting from nerve cuff stimulation and manual whisker deflection. $a$, Schematic diagram of nerve cuff electrode and microwire placement. $b, c$, Peristimulus time histograms of responses to nerve cuff stimulation and manual whisker deflection from the same recording session in anesthetized rats. Examples of a single-unit and a multiunit recording are shown for VPM $(c)$ and SI $(b)$. Note that $b$ and $c$ were collected from two separate animals. Vertical dashed lines indicate the time of stimulus presentation $(0 \mathrm{msec})$.

implanted as one comb-like unit (see Nicolelis et al., 1997). As with the thalamic penetrations, neural activity was recorded and receptive fields were mapped while the electrodes were being lowered into the SI cortex to monitor their location. Once in place, the electrode array was also cemented to the skull and screws.

During the same surgery, a cuff electrode was implanted around the infraorbital branch of the trigeminal nerve, just rostral to the infraorbital 
fissure and contralateral to the implanted microwires. A dorsoventral incision was made on the face lateral to the infraorbital nerve. Tissue was dissected until the nerve was exposed and perineurium was cleared away so a piece of surgical silk could be inserted under the nerve. The cuff electrode was then drawn around the nerve until it lay inside the cuff. The cuff was tied closed around the nerve using the piece of surgical silk imbedded in the Sylgard on the outer edge of the cuff. Wires to the electrode were led subcutaneously to a connector on the skull.

After the microelectrode and cuff electrode implantations, the surgical incisions were sutured, and antibiotic ointment was applied to the wounds. An analgesic (Buprenex; $0.1 \mathrm{mg} / \mathrm{kg}$ ) was administered via subcutaneous injection after the surgery and for $3 \mathrm{~d}$ after surgery. Animals were allowed to recover for 1 week before recording sessions commenced.

Recording procedures. The simultaneous, multisite neural ensemble recording techniques used in this paper have been described in detail elsewhere (Nicolelis et al., 1997). Briefly, neural recordings were obtained using chronically implanted stainless steel microwires. Bundles of 16 wires were used for VPM implants, and arrays of 16 wires were used for cortical implants. A many neuron acquisition processor system (Plexon, Dennison, TX) was used to process the signals from the microwires on-line. Waveforms were discriminated using pairs of voltage time windows to isolate individual waveforms. The time stamps of these waveforms were then stored on a personal computer and used to create the peristimulus time histograms used for the analyses.

Stimulation protocol. The infraorbital nerve was stimulated via the nerve cuff electrode throughout each of the recording sessions. Stimuli were generated using a Grass S8800 stimulator in conjunction with a Grass PSIU6 stimulus isolation unit. The stimulation strength (i.e., the current level) was determined individually for each rat during the first experiment session by measuring the response to stimulation throughout a range yielding no tactile responses through robust tactile responses. The testing range was $4-11 \mathrm{~mA}$. The magnitudes of the responses were assessed by creating peristimulus time histograms (PSTHs) and measuring the magnitude of the peak of the response to the stimulus. The stimulation level was set $1 \mathrm{~mA}$ higher than the threshold that just produced minimal neural responses in a given rat (as indicated by the PSTHs), and this level was used in all subsequent experiments involving that rat. The average current level used was $7.0 \mathrm{~mA}$ (range, 5-9 mA). In all cases, there was a narrow range, typically 1-2 mA, over which responses appeared. Below this threshold, there were no thalamic or cortical responses to cuff stimulation, and above it, increasing the stimulation strength only increased the magnitude of the responses somewhat but generally not the number of channels that responded to the stimulation. Each stimulus was $100 \mu \mathrm{sec}$ in duration. Animals never demonstrated discomfort when stimuli were presented at these levels.

We demonstrated that stimulation provided via the nerve cuff electrode was consistent throughout the behavioral states described in this paper by recording from a hook electrode proximal to the implanted cuff electrode on the infraorbital nerve in anesthetized animals (data not shown). We stimulated the nerve with $100 \mu \mathrm{sec}$ pulses at a rate of $0.2 \mathrm{~Hz}$ throughout a range of current levels. The voltage recorded with the hook electrode in response to the cuff stimulation was recorded using a digital oscilloscope (HM407; Hameg, East Meadow, NY). We compared the magnitudes of the compound action potentials recorded from the hook electrode in response to nerve stimulation while whiskers were (1) in their resting, fully retracted position, (2) held in a fully protracted position, and (3) manually deflected at a rate of $2 \mathrm{~Hz}$. There were no statistically significant differences in the size of the compound action potentials between these three states. These results confirmed that there was no change in the magnitude of the response evoked by nerve cuff stimulation when the whiskers were moved or held in their fully protracted position. In addition, we implanted nerve cuff electrodes in several animals and videotaped the electrode during manual deflection of the whiskers. In all cases, no relative movement between the nerve and the cuff electrode was observed, indicating that the cuff remained in a constant position relative to the nerve regardless of whisker position. These results confirm those of Grill and Mortimer (1998) in a study recording from the cat sciatic nerve demonstrating that chronically implanted nerve cuff electrodes do not move relative to the nerve around which they are implanted and that uniform amounts of current are delivered regardless of movement that may occur near the electrode (Grill and Mortimer, 1998).

Throughout each recording session, stimuli were presented every 2 sec, either individually or in pairs of two stimuli. Two seconds were chosen as the interval between single stimuli, as well as between pairs of stimuli, because this provided sufficient time for the neural activity to return back to baseline levels after a stimulus (data not shown). The individual stimuli presented $2 \mathrm{sec}$ apart will be referred to as "single stimuli" throughout this report. When pairs of stimuli were presented, the interstimulus interval was held constant during a given recording session but was varied between recording sessions. The interstimulus intervals for stimulus pairs used in these experiments were 25, 50, 75, 100, 125, 150, 175, and $200 \mathrm{msec}$. Electrical stimuli delivered via the cuff electrode produced responses in VPM and SI that were very similar to those obtained by mechanical deflection of individual whiskers (see Fig. 1b,c), as in Nicolelis et al. (1997). The main difference we observed was that response latencies were shorter after the cuff stimulation than after mechanical whisker deflection.

Recording sessions with awake animals. Animals were placed in a well lit recording chamber $(60 \mathrm{~cm}$ long and $30 \mathrm{~cm}$ wide), open on one end for videotaping purposes, and allowed to move freely during the entire recording session. The cables for neural recordings were connected to a rotating commutator on the top of the chamber to minimize interference with the animal's movements. Electrical stimulation was delivered to the infraorbital nerve cuff electrode throughout a given recording session according to the protocols described above. Recording sessions lasted $1 \frac{1}{2}-2 \mathrm{hr}$. Throughout an entire recording session, the animals were videotaped using a high-resolution video camera recording 60 frames per second. A time stamp (resolution, $1 \mathrm{msec}$ ) that was synchronized with the neural recordings was put on each frame of the videotape (one every 17 msec because of the $60 \mathrm{~Hz}$ camera resolution) to allow precise correlation of the animal's behavior with the recorded neural activity. Up to two recording sessions were performed on a given day, and at least $1 \mathrm{~d}$ of rest was allowed between recording days.

Recording sessions with anesthetized animals. Each rat underwent one recording session under pentobarbital anesthesia. This was typically the last recording session performed. Rats were first briefly anesthetized with halothane $(\sim 1 \mathrm{~min})$ and then given an injection of sodium pentobarbital $(50 \mathrm{mg} / \mathrm{kg}$, i.p. $)$. Anesthesia was maintained with supplemental injections of pentobarbital $(0.05 \mathrm{cc})$ administered when the animal's breathing rate increased and/or the animal became responsive to foot pinch. Stimulation of the infraorbital nerve was presented via the nerve cuff electrode using the same stimulus protocols as with the awake animals except that individual stimuli or pairs of stimuli with a given interstimulus interval were presented in blocks of $600 \mathrm{sec}$ (300 stimuli total).

Data analysis. Videotapes of the recording sessions were analyzed to identify the different behavioral states (see below) of the animal throughout the recording session. Time epochs during which each of the four target behavioral states occurred were identified using the time stamps on the video frames. Subsequently, stimuli were sorted into four groups corresponding to the behavioral state during which they had been presented. Stimuli were only used for analysis if the behavioral epoch during which they occurred lasted for $6 \mathrm{sec}$ or more (i.e., stimuli for a given behavior were only accepted if they occurred in groups of three or more). Periods of the videotape during which the rat's behavior either did not fit in one of the behavioral categories (e.g., grooming), was ambiguous (e.g., slight, arrhythmic whisker movements), or was not observable (e.g., rat not facing camera) were discarded from the analysis.

Four behavioral states were defined for this study. (1) Quiet immobility: rat awake and standing or sitting still, showing no voluntary motor activity. During this state, animals had their eyes fully open, and their heads were not resting on the substrate. (2) Active: rat showing voluntary motor activity not including movement of the whiskers. This usually occurred during exploratory activity (e.g., walking and/or inspecting objects) and could include head movements, jaw movements, and/or movement of the limbs or trunk. (3) Whisking: large-amplitude sweeps of the whiskers (ends of the whiskers moved several centimeters) at a rate of $\sim 4-6 \mathrm{~Hz}$, either across objects or in the air. This was also often associated with exploratory or locomotor activity. Often, this behavior was encouraged by introducing items such as blocks into the recording chamber. (4) Whisker twitching: small-amplitude whisker movements (ends of whiskers moved a couple of millimeters) at a rate of 7-12 Hz, concurrent with $7-12 \mathrm{~Hz}$ oscillations of neural firing in SI and VPM. This behavior usually occurred during periods in which the animal was standing or sitting still. Although animals in this state were immobile except for whisker movements, they were able to respond to sensory stimuli and were easily disrupted by auditory, tactile, or visual stimulation. The 7-12 $\mathrm{Hz}$ oscillations that preceded and accompanied whisker-twitching movements were identified by listening to the output of an audio monitor 
a single unit recordings

\section{quiet}
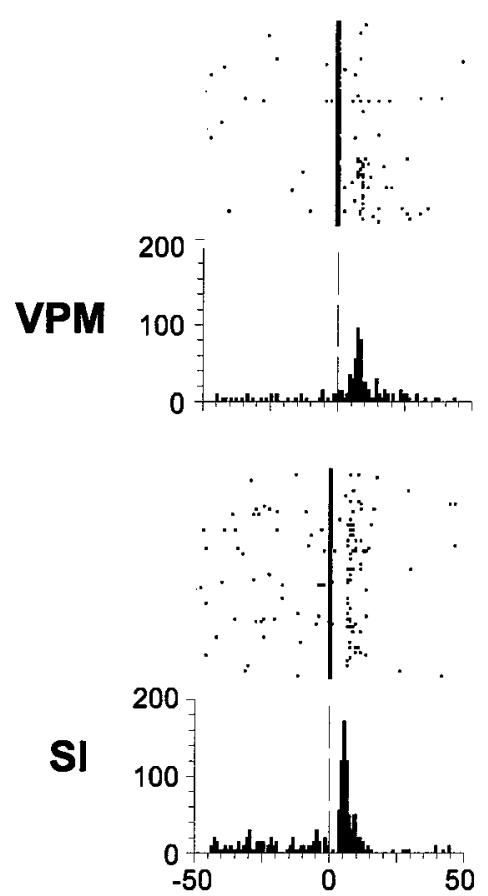

b

multiunit recordings

quiet
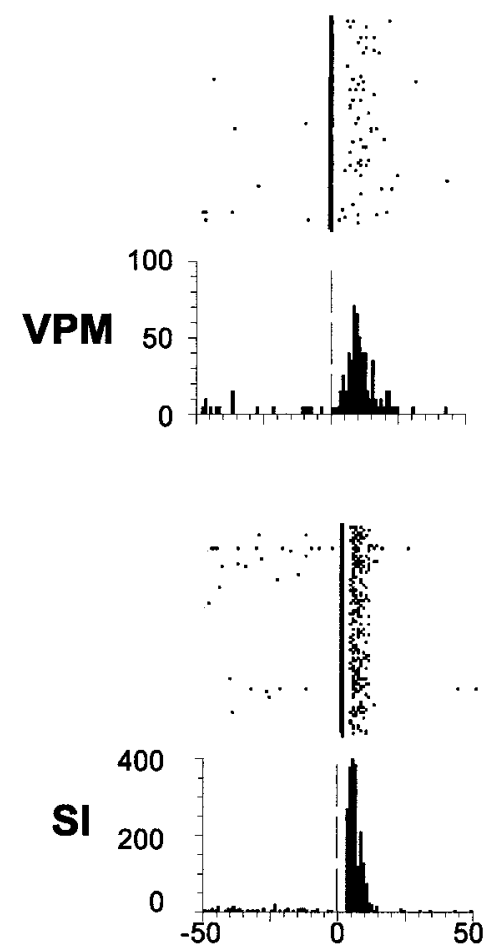

active
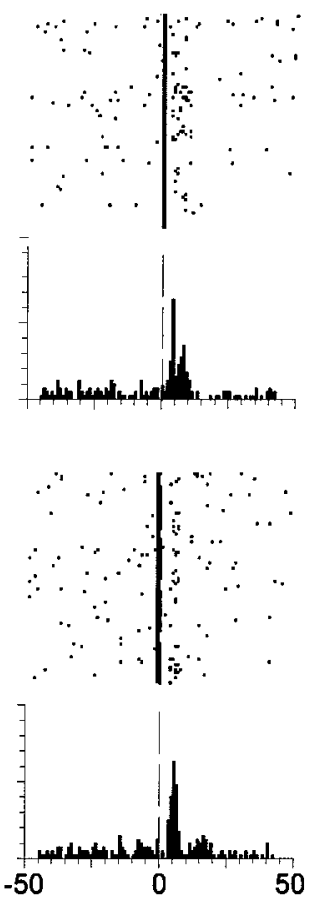

active
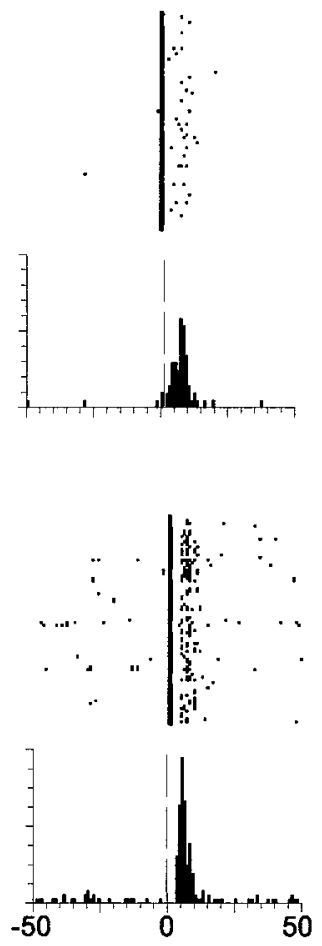

whisking
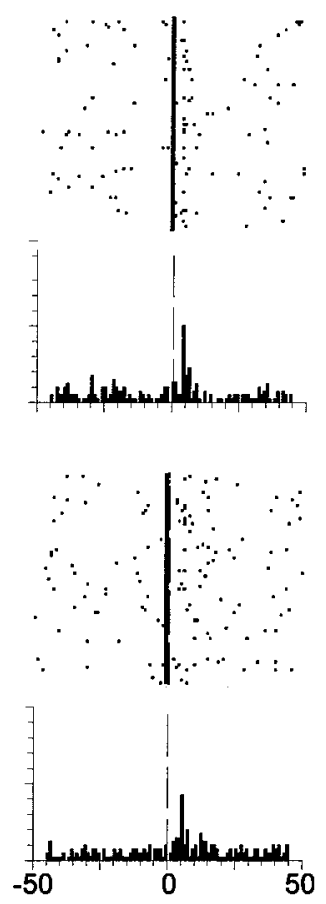

whisking
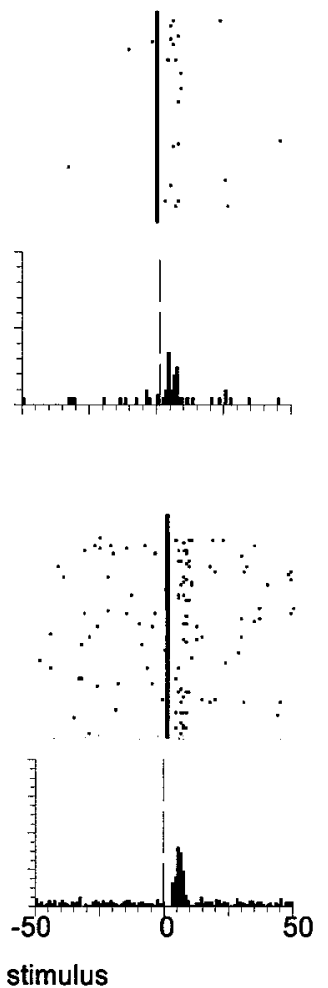

whisker twitching
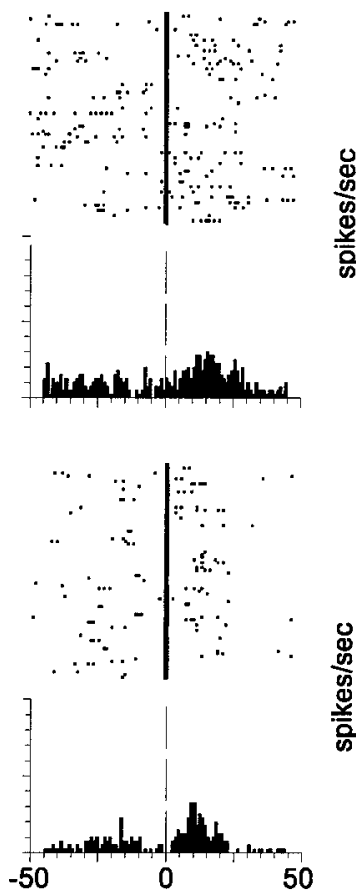

whisker twitching
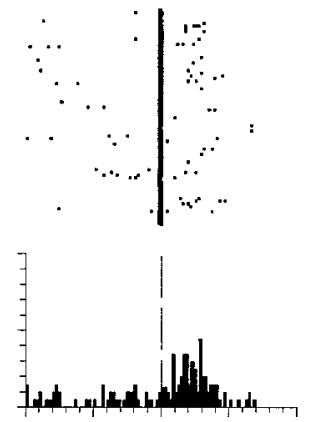

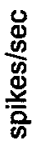

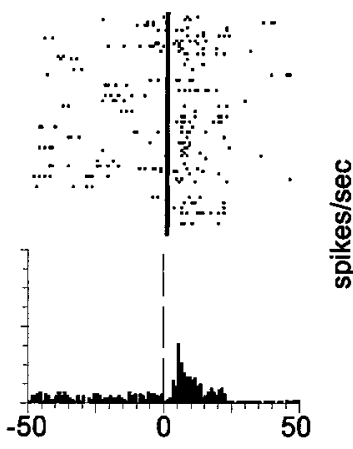

Figure 2. Single-unit and multiunit responses to individually presented tactile stimuli during different behavioral states. Peristimulus time histograms (bottom) and the accompanying raster plots (top) were each compiled from 200 stimulus presentations. Vertical dashed lines in the histograms and vertical solid lines in the raster plots represent the time of stimulus presentation $(0 \mathrm{msec})$. $a$, Responses of a single unit in VPM and a single unit in SI during four behavioral states. $b$, Responses from a multiunit recording in VPM and one in SI during four behavioral states. 
signal depicting ongoing multiunit neural activity that was recorded on the videotape. The relationship between these oscillations and the whisker-twitching movements have been described previously (Semba and Komisaruk, 1984; Nicolelis et al., 1995).

Quantification of neural signals. Raster plots and PSTHs were generated for responses on each channel to stimulation of the nerve cuff electrode. These histograms were created using 200 stimuli for each condition and had a bin size of $1 \mathrm{msec}$. In all analyses, the beginning of the time period used was set at $2 \mathrm{msec}$ to eliminate the electrical artifact that could be observed in the $0-1 \mathrm{msec}$ time bin on some channels resulting from cuff stimulation. The peaks in the PSTHs corresponding to short-latency excitatory responses to the stimuli were assessed in two ways. First, the maximum height of the peak was measured (referred to as "maximum magnitude"). Second, the total amount of firing during a period from 2 to $20 \mathrm{msec}$ after the stimulus was calculated. This second measure was used to assess the overall amount of response a stimulus yielded, because some responses differed not only in magnitude but also in width and intensity across the experimental conditions. This second measure will be referred to hereafter as the "integrated response." For both of these measures, the background firing rate was calculated for each channel (average firing rate during the $100 \mathrm{msec}$ period preceding the stimulus) and subtracted from the response. Both of these measures were normalized to the quiet state activity levels. For pairs of stimuli, responses were normalized to the first stimulus of pairs presented during the quiet behavioral state. Responses were compared using ANOVAs, and Tukey's honestly significant difference (HSD) tests were used when post hoc tests were needed. A response was considered significant if the $p$ value was $<0.05$. Only one recording session was conducted for each stimulation protocol in each rat, ensuring that no neurons that could potentially have been recorded from in multiple sessions were statistically pooled.

Overall levels of neural activity before and after short-latency excitatory responses to stimuli were assessed by averaging histograms across all channels used in the analyses for a given recording area (VPM or SI). The levels of neural activity before a stimulus were calculated by averaging the number of spikes per second during the $100 \mathrm{msec}$ period preceding stimulus presentation. Spiking activity was also calculated at points every $25 \mathrm{msec}$ after the stimulus.

Histology. After the entire battery of experiments was completed (usually 3-4 weeks after electrode implantation), rats received an overdose of sodium pentobarbital and were then perfused transcardially with $0.9 \%$ saline, followed by $5 \%$ formalin. The brains were sliced into $80 \mu \mathrm{m}$ coronal sections and stained using cresyl violet. In these sections, the location of the tips of the electrodes could be determined, and their locations in the whisker representations of VPM and SI were verified.

Parts of this paper have been published previously (Fanselow et al., 1997).

\section{RESULTS}

To evaluate the effects of different behavioral states (quiet, active, whisking, and whisker twitching) on the somatosensory responses of VPM and SI neurons, both single-unit and multiunit recordings were made during the experiments described here. On-line and off-line waveform measurements, as well as off-line analysis of interspike interval histograms, were used to identify single units in our records. A recording was identified as a single unit if it met all three of the following criteria: (1) the presence of reproducible waveforms that could be isolated by our on-line recording procedure (Nicolelis et al., 1997), (2) interspike interval histograms showing that $<5 \%$ of the interspike intervals were shorter than $1.2 \mathrm{msec}$, and 3) the negative-going component of the waveform for each action potential being at least $75 \mu \mathrm{V}$ in amplitude (average background noise levels were 15-20 $\mu \mathrm{V}$, so the signalto-noise ratios were 4-5). According to these criteria, we recorded a total of 62 single units in the VPM thalamus and 61 in the SI cortex in five awake, freely behaving rats. The other signals obtained from these animals during the same experimental sessions were designated as multiunit recordings. A total of 165 multiunit recordings were made in the VPM thalamus, and 157 were made in the SI cortex. Because the rat VPM thalamus contains only a single cell type (thalamocortical-projecting neurons), our multiunit data likely derived from a rather homogenous population of neurons in this structure. For some of the statistical analyses in this paper, we decided to group single-unit and multiunit recordings within each area. This decision was validated by the fact that our single-unit results were similar to the findings obtained with multiunit data (see Figs. 2, 3).

In each animal, after $\sim 4$ weeks of recording, a final recording session was performed under pentobarbital anesthesia. Because these recordings were performed several weeks after microwire implantation, the neuronal yield was typically lower than in earlier recording sessions. Therefore during the experiments with anesthetized animals, a total of 25 single units were recorded in the VPM thalamus, and 11 were recorded in the SI cortex; the total numbers of multiunit recordings in VPM and SI were 56 and 47 , respectively.

We compared cortical and thalamic somatosensory responses produced by electrical stimulation of the nerve cuff electrode with those triggered by mechanical deflection of individual facial whiskers. This was done by stimulating the infraorbital nerve via the cuff electrode at $1 \mathrm{~Hz}$ in anesthetized animals and comparing the responses of SI (Fig. 1b) and VPM (Fig. 1c) neurons with those obtained via $1 \mathrm{~Hz}$ mechanical displacement of individual whiskers. Whiskers were mechanically displaced in discrete steps (100 msec duration) using a motor-driven manipulator that was placed next to each whisker individually and activated by a Grass S8800 stimulator. This analysis revealed that the parameters for electrical stimulation of the infraorbital nerve used in this study (see Materials and Methods) could produce sensory responses very similar to those obtained by mechanical whisker deflection (for examples, see Fig. 1b,c). Cortical and thalamic sensory responses to cuff stimulation were of similar shape and amplitude to those obtained by manual whisker deflection. Electrical nerve stimulation did, however, induce faster sensory responses in both VPM thalamus and SI cortex. In addition, these control experiments revealed that the nerve cuff could be used for long periods of time after implantation and that reproducible stimuli could be delivered chronically. Our results are consistent with data obtained using a similar cuff electrode design to stimulate the optic nerve (Weliky and Katz, 1997).

\section{Responses to single stimuli}

Our first step was to quantify the sensory responses of both VPM and SI neurons when individual stimuli were presented (i.e., one stimulus every $2 \mathrm{sec}$ ) during the four different behavioral states described above. The peristimulus time histograms in Figure 2 show examples of responses to stimuli presented during each behavioral state investigated, for both single-unit and multiunit recordings. Average data for five animals are shown in Figure 3, $a$ (single units) and $b$ (single unit and multiunit combined). In this figure, both the average maximum magnitude and the average integrated responses are illustrated for each of the four behaviors.

Quantitative analysis of these data revealed that neuronal response levels (spikes per second) recorded simultaneously in VPM and SI during the quiet and active behavioral states were not significantly different from one another. In contrast, responses to sensory stimuli presented during either type of whisker movement (whisking or whisker twitching) were decreased in both the VPM thalamus and SI cortex, compared with that in the quiet state (see Fig. 3). For single-unit recordings, there were statistically significant reductions compared with that in the quiet state under the following conditions: VPM integrated response, whisk- 

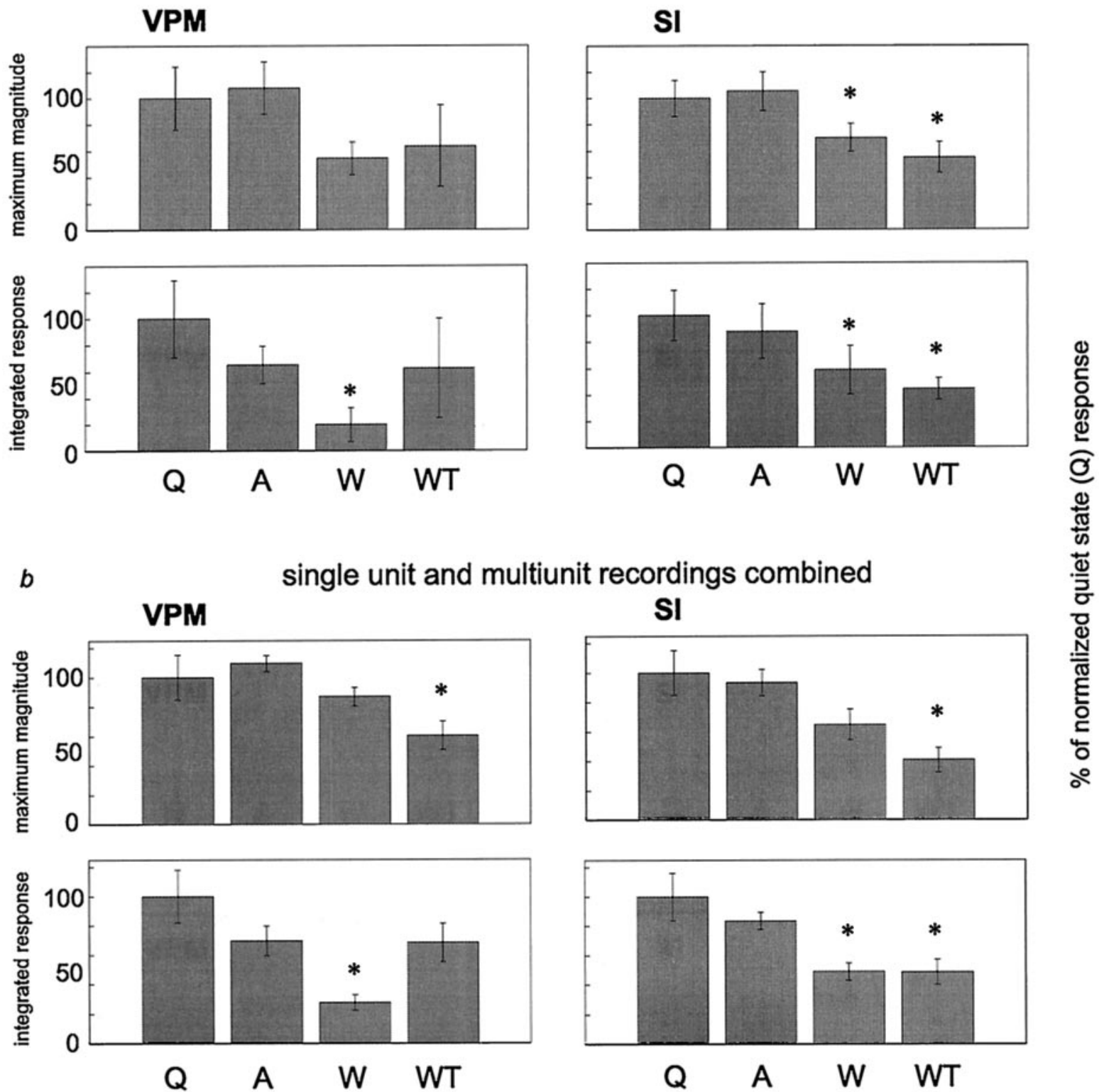

Figure 3. Average responses to single stimuli during different behavioral states. In each graph, responses were normalized to responses during the quiet state. $a$, Average responses of single units. $b$, Average responses of single-unit and multiunit recordings combined. Top graphs in $a$ and $b$ represent the maximum magnitude (max mag) of the responses. Bottom graphs in $a$ and $b$ represent the integrated response (intg) values (see Materials and Methods for a description of these measures). Asterisks indicate values significantly different from the quiet state in each graph. Error bars represent \pm SEM. ANOVA results: $a$, VPM max mag, $F=1.99, p=0.187$; VPM intg, $F=8.77, p=0.0012$; SI $\max \operatorname{mag}, F=5.74, p=0.0022$; SI intg, $F=10.99, p=$ 0.000023. $b$, VPM max mag, $F=9.82, p=0.0099$; V PM intg, $F=10.22, p=0.0090 ;$ SI $\max \operatorname{mag}, F=9.82, p=0.0099 ;$ SI intg, $F=23.94, p=0.00097$. Please see text for $p$ values from post hoc tests (Tukey's HSD). A, Active; $Q$, quiet; $W$, whisking; $W T$, whisker twitching.

ing $(-80.3 \pm 13.1 \% ; p<0.001)$; SI maximum magnitude, whisking $(-30.0 \pm 10.7 \% ; p<0.05)$ and whisker twitching $(-45.1 \pm$ $11.7 \% ; p<0.02)$; and SI integrated response, whisking $(-41.29 \pm$ $18.3 \% ; p<0.001)$ and whisker twitching $(-55.6 \pm 8.3 \% ; p<$ $0.001)$. For the pooled single-unit and multiunit data, there were statistically significant reductions observed in the following cases: VPM maximum magnitude, whisker twitching $(-39.7 \pm 9.7 \%$; $p<0.02)$; VPM integrated response, whisking $(-72.5 \pm 5.4 \%$; $p<0.01)$; SI maximum magnitude, whisker twitching $(-53.22 \pm$ $8.4 \% ; p<0.02)$; and SI integrated response, whisking $(-51.2 \pm$
$5.8 \% ; p<0.002)$ and whisker twitching $(-48.5 \pm 8.4 \% ; p<$ $0.005)$.

\section{Magnitude of neuronal activity before and after stimulus presentation}

The demonstration that the magnitude of sensory neuronal responses varied according to the animal's behavior led us to examine whether further variations in firing rate could be observed before and after the stimulus and initial excitatory response. This analysis revealed that the background levels of firing 

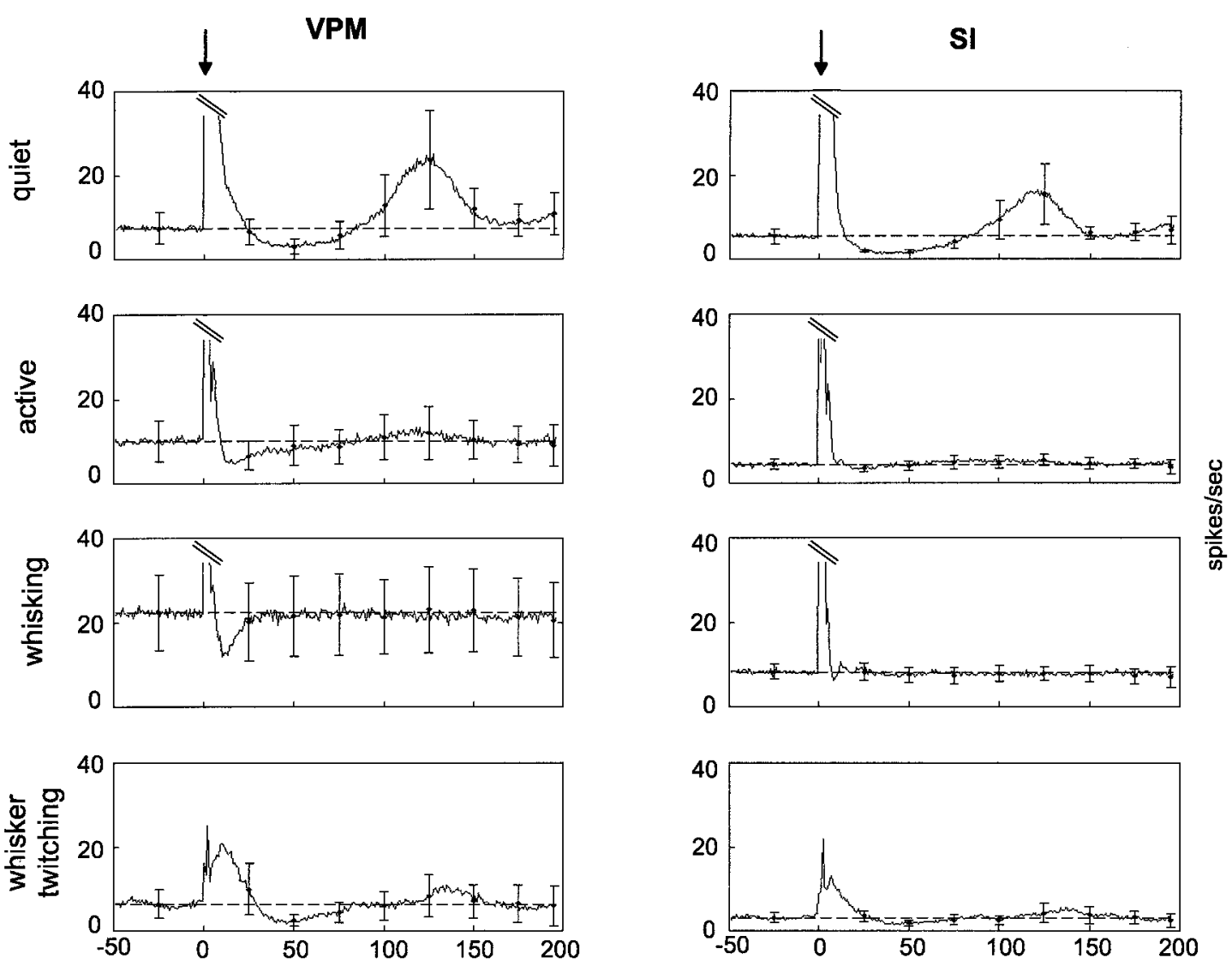

ms post stimulus

Figure 4. Average levels of neural activity before and after presentation of single stimuli across four behavioral states. Traces are averages of 200 trials from each of five rats (quiet, active, and whisking) and three rats (whisker twitching) and include single-unit and multiunit data combined. Arrows above each column represent stimulus onset $(0 \mathrm{msec})$. The $y$-axes are the same across all four behavioral conditions, necessitating truncation of the response magnitudes ( parallel diagonal lines) in quiet, active, and whisking. Horizontal dashed lines in each graph show the average baseline level, calculated as the average activity level in the $100 \mathrm{msec}$ preceding stimulus onset. Error bars represent \pm SEM of points $25 \mathrm{msec}$ before and 25, 50, 75, 100, 125, 150, 175, and $200 \mathrm{msec}$ after the stimulus. Left, Responses in VPM. Right, Responses in SI. Note that the truncated peaks in this figure only include neural responses to the stimulation and do not include electrical artifact resulting from cuff stimulation. Stimulation artifact occurred in the 0 msec time bin and was removed when plotting these figures by excluding that bin.

before the presentation of a stimulus differed from one behavioral state to the next. The average prestimulus firing rate values in spikes per second (averaged over the $100 \mathrm{msec}$ before the stimulus) for single units in VPM were: quiet, $1.3 \pm 0.2$; active, $2.7 \pm$ 0.7; whisking, $4.2 \pm 0.9$; and whisker twitching, $5.8 \pm 0.5$. In SI, the single-unit values were: quiet, $2.3 \pm 0.4$; active, $2.7 \pm 0.5$; whisking, $3.6 \pm 0.7$; and whisker twitching, $2.0 \pm 0.3$. In VPM thalamus, the prestimulus single-unit firing levels during the active, whisking, and whisker-twitching states were significantly higher than that during the quiet state $(p<0.01)$. In SI, the prestimulus single-unit firing rate during whisking was significantly higher than that during the quiet and whisker-twitching states $(p<0.02)$. We also calculated average prestimulus firing rates using both the single-unit and multiunit recordings combined (see Fig. 4 before stimulus presentation at $0 \mathrm{msec}$ ) and found similar results overall, although the values for the combined analysis were larger in magnitude, as would be expected when including the multiunit recordings in which more neurons were recorded per channel. The combined values in VPM in spikes per second were: quiet, $7.5 \pm 3.7$; active, $9.8 \pm 4.9$; whisking, $21.3 \pm$ 9.0; and whisker twitching, $5.8 \pm 3.4$. In SI, the average firing rate values were: quiet, $5.5 \pm 1.8$; active, $4.3 \pm 1.2$; whisking, $8.1 \pm 1.8$; and whisker twitching, $3.0 \pm 1.3$. In VPM, the multiunit firing level during the whisking state was significantly higher than that in the quiet state $(p<0.01)$. In SI, the prestimulus multiunit firing rate during whisking was significantly higher than that during the active state $(p<0.03)$.

Firing rates after the presentation of a stimulus also varied in several ways according to the animal's behavior. First, the duration of the initial excitatory sensory response varied according to the animal's behavioral state (Fig. 4). During the quiet state, the initial excitatory responses lasted $\sim 24 \mathrm{msec}$ in the VPM thalamus and $15 \mathrm{msec}$ in the SI cortex. During episodes of whisker twitching, the excitatory responses lasted longer, $\sim 33 \mathrm{msec}$ in VPM and $25 \mathrm{msec}$ in SI. In contrast, the excitatory responses during the active and whisking states were substantially shorter, lasting 8-10 msec in both areas.

The firing rate after the first excitatory response differed according to behavioral state in both VPM thalamus and SI cortex (Fig. 4). During the quiet state, the period after stimulus presentation showed a characteristic epoch of reduced firing, compared with prestimulus baseline levels, which has been described by other researchers. This period of reduced firing in VPM and SI during the quiet state lasted for $\sim 50 \mathrm{msec}$. In contrast, during the 
a

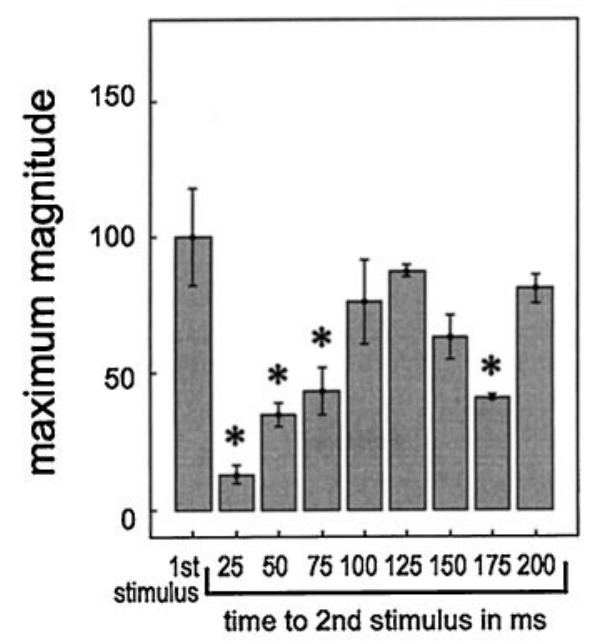

b

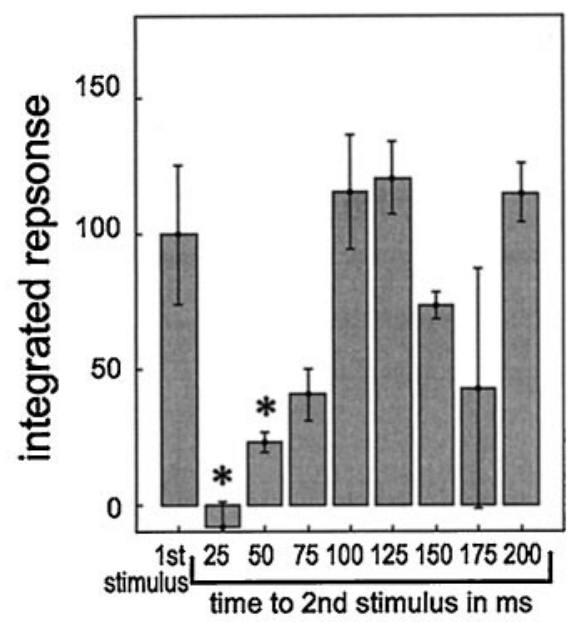

Active
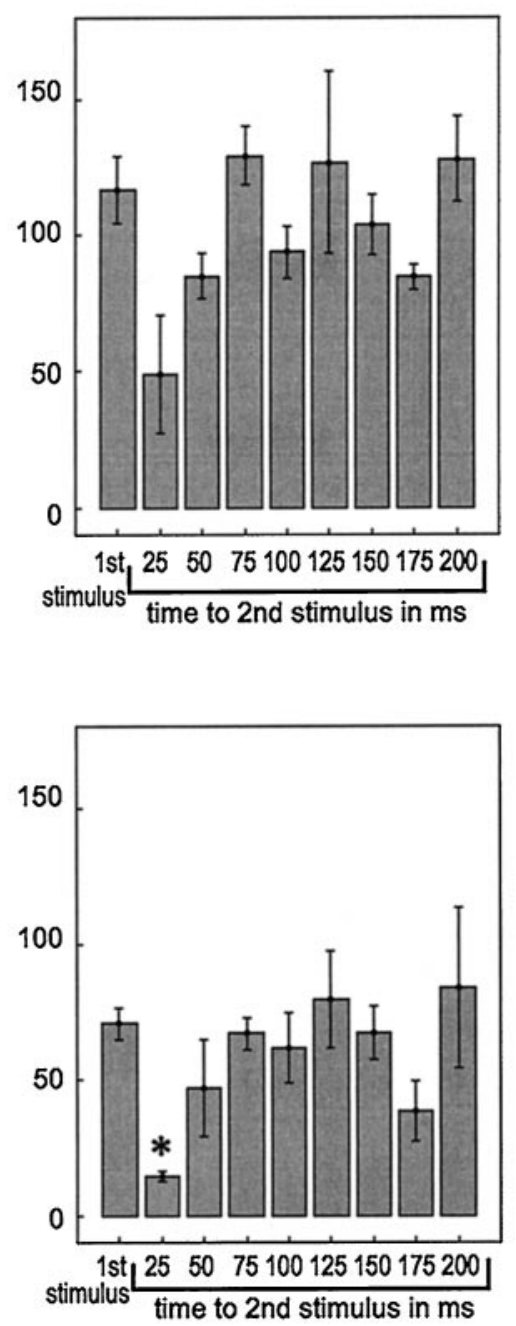

Whisking
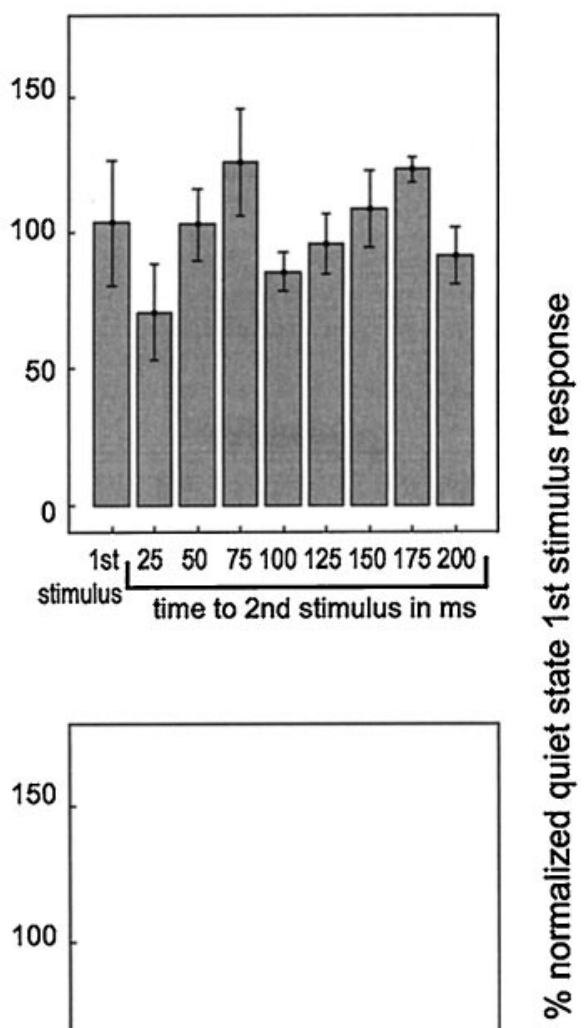

0

1st. 255075100125150175200

Figure 5. Responses in VPM to pairs of stimuli with varying interstimulus intervals during different behavioral states. In each graph, average responses are shown to the first stimulus in a pair (left-hand bar on each graph labeled "1st stimulus") and to the second stimulus in the pairs with varying interstimulus intervals (interstimulus interval shown below bar). All responses were normalized to responses to the first stimulus in the pairs during the quiet state. These data represent single-unit and multiunit data combined. Error bars represent \pm SEM, and asterisks represent values that are significantly different from the responses to the first stimulus in the pairs within each behavioral state. $a$, The maximum magnitude of the responses. $b$, The integrated response values. ANOVA results: $a$, quiet, $F=10.42, p=0.000004$; active, $F=2.49, p=0.04$ (no differences found between the first stimulus and any other values); whisking, $F=1.20, p=0.34$. $b$, quiet, $F=9.90, p=0.000006$; active, $F=2.71, p=0.03$; whisking, $F=0.3, p=0.96$. Please see text for $p$ values from post hoc tests (Tukey's HSD).

active and whisking states, this postexcitatory period of reduced firing was substantially shorter and, in most cases, yielded less spike suppression. During the active behavioral state, there was a $35 \mathrm{msec}$ period of reduced activity in the VPM thalamus, whereas no such period was detected in the SI cortex. Likewise, during whisking, there was a $20 \mathrm{msec}$ period of reduced firing in the VPM thalamus but none in the SI cortex. During episodes of whisker twitching, the period of suppressed firing $(\sim 50 \mathrm{msec}$ in duration) in VPM was much more evident than in the SI cortex. This postexcitatory reduction of firing ended at approximately the same poststimulus time in both the quiet and the whisker- twitching states. However, the beginning of this period was delayed $10 \mathrm{msec}$ in the latter state.

Finally, during quiet immobility, a pronounced long-latency excitatory response component was observed in both VPM and SI. These long-latency responses started at $\sim 82 \mathrm{msec}$ after the stimulus presentation in both VPM and SI, peaked at $\sim 120 \mathrm{msec}$ after the stimulus in both structures, and lasted until $175 \mathrm{msec}$ after the stimulus in the VPM thalamus and $150 \mathrm{msec}$ after the stimulus in the SI cortex. Minimal traces of such long-latency responses were observed during the active and whisker-twitching states in VPM. However, no long-latency components were ob- 


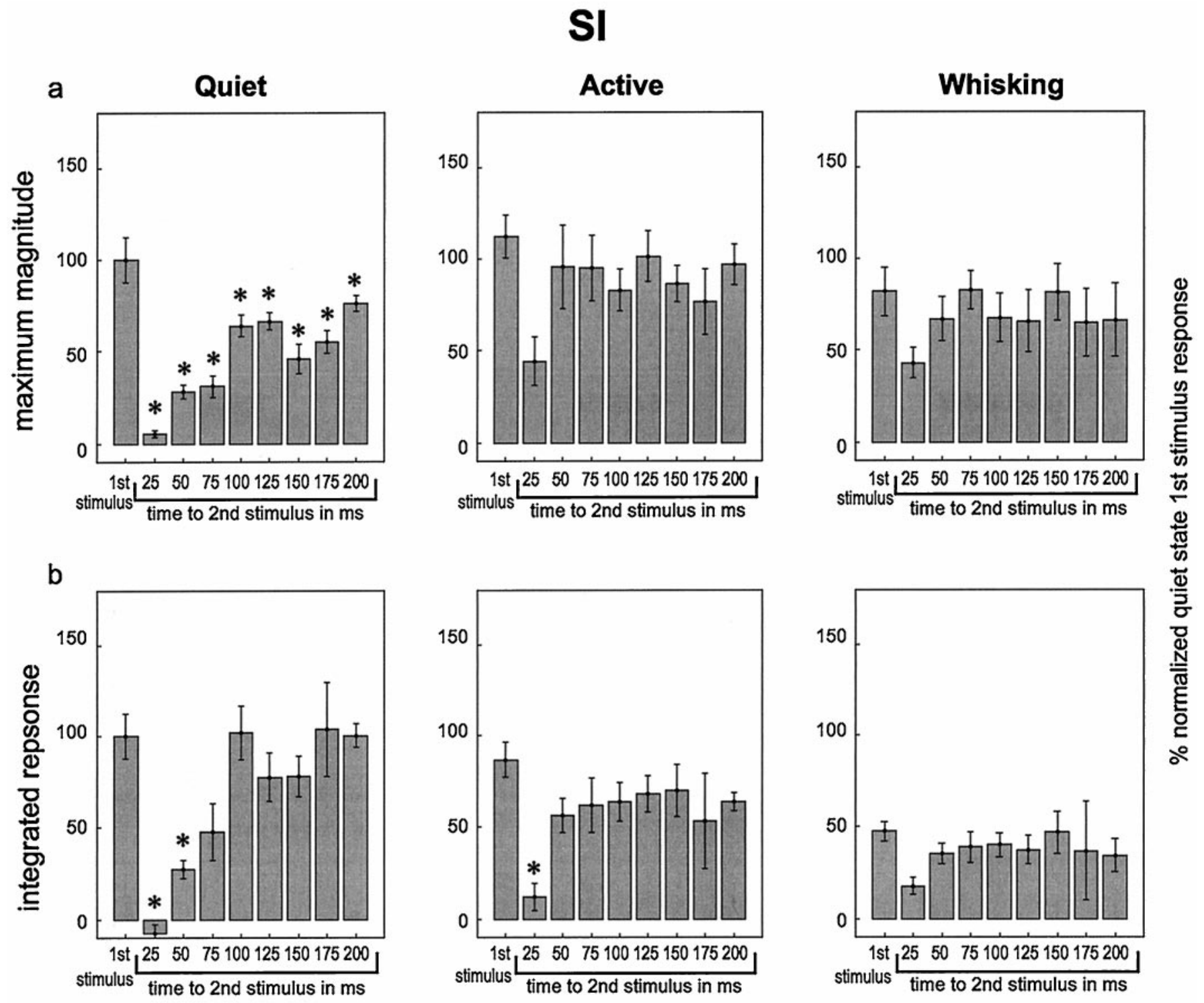

Figure 6. Responses in SI to pairs of stimuli with varying interstimulus intervals during different behavioral states. Labeling conventions are described in Figure 5. ANOVA results: $a$, quiet, $F=18.9, p<0.000001$; active, $F=1.86, p=0.11$; whisking, $F=0.73, p=0.66 . b$, quiet, $F=11.95, p=0.000001$; active, $F=3.70, p=0.006$; whisking, $F=1.17, p=0.39$. Please see text for $p$ values from post hoc tests (Tukey's HSD).

served in cortical recordings in any state other than the quiet state.

\section{Responses to pairs of stimuli}

We next wanted to determine whether different behavioral states also influenced the magnitude and latency of neuronal sensory responses during presentation of pairs of stimuli at different interstimulus intervals (25-200 msec). Previous studies have demonstrated that when one stimulus closely follows another, there is a reduction in the response magnitude of the second stimulus (Laskin and Spencer, 1979; Simons, 1985; Simons and Carvell, 1989; Castro-Alamancos and Connors, 1996b), and that the reduction in the response magnitude of the second stimulus is correlated with the period of suppressed firing observed after the first stimulus (Simons, 1985; Simons and Carvell, 1989). Our observation that the duration of the period of reduced firing after a single stimulus varied according to behavioral state suggested to us that the magnitude of cortical and thalamic sensory responses to a second stimulus could also be behavior dependent.

To test this hypothesis, we delivered pairs of stimuli with varying interstimulus intervals to assess how the amount of time between the stimuli impacted the magnitude of the response to the second stimulus. Figures 5 and 6 summarize the results obtained in these experiments. In each graph within these figures, the left-hand bar represents the response to the first stimulus in the pair, averaged across all interstimulus intervals. The other bars show the magnitude of the response to the second stimulus, presented at the interstimulus interval listed below the bar. Several observations were derived from these experiments. First, during the quiet state, the neuronal responses in both VPM and SI to the second stimulus in the pair were significantly smaller than the neuronal responses to the first stimulus for interstimulus intervals of 25,50 , or $75 \mathrm{msec}$. The reduction in response to the second stimulus was attenuated (i.e., the responses grew larger) as 
a

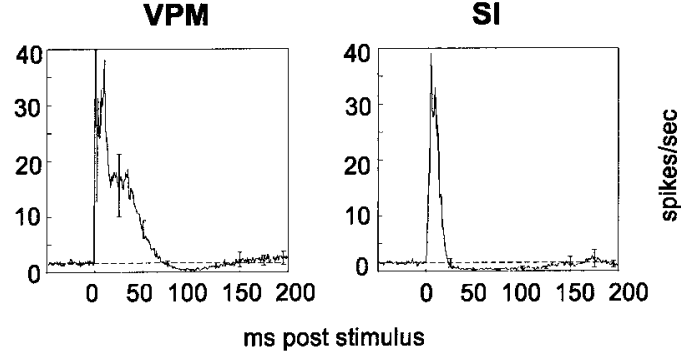

$b$
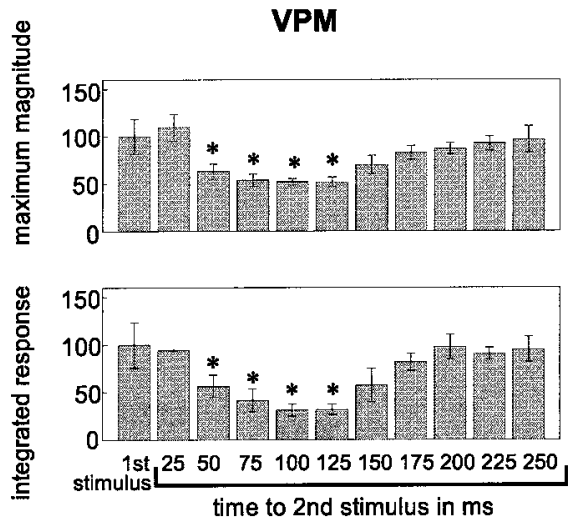

$c$
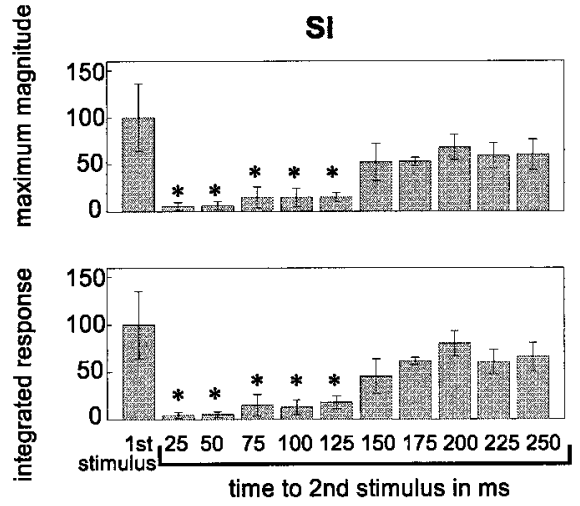

Figure 7. Sensory responses during pentobarbital anesthesia. $a$, Average levels of neural activity in VPM and SI before and after stimulus presentation. Labeling conventions for $a$ are described in Figure 4. $b, c$, Response magnitude and integrated response to pairs of stimuli at varying interstimulus intervals. Labeling for $b$ and $c$ is described in Figure 5. These data represent single-unit and multiunit data combined. ANOVA results: $b$, $\max \operatorname{mag}, F=6.45, p=0.003$; intg, $F=7.12, p=0.002 ; c$, $\max$ mag, $F=4.44, p=0.014$; intg, $F=6.02, p=0.004$. Please see text for $p$ values from post hoc tests (Tukey's HSD).

the interstimulus interval increased until the interstimulus interval reached $100 \mathrm{msec}$, at which point the response to the second stimulus in the pair was not statistically different from the first in VPM but remained slightly (and statistically significantly) below the first stimulus values in SI throughout the $200 \mathrm{msec}$ interstimulus intervals tested in this study. This effect mirrored the timing and duration of the period of postexcitatory firing suppression observed in VPM and SI (see Fig. 4). Interestingly, the response suppression effect returned at $150-175 \mathrm{msec}$ interstimulus intervals during the quiet state (see maximum magnitude plots in Figs. 5,6 ), a period during which neuronal firing in the VPM thalamus and SI cortex tended to return to baseline levels after the presentation of individual stimuli (see Fig. 4). a

\section{latency for single stimuli}

VPM

SI
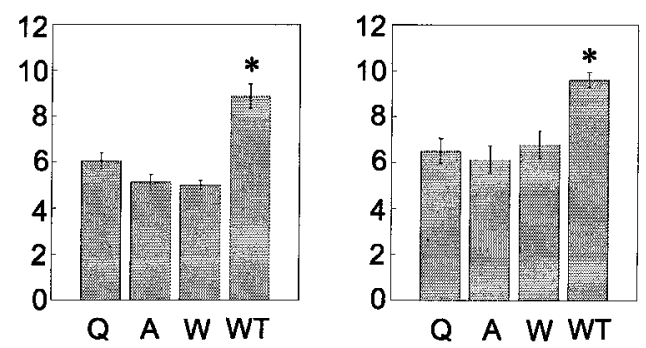

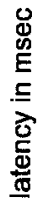

b

latency for paired stimuli

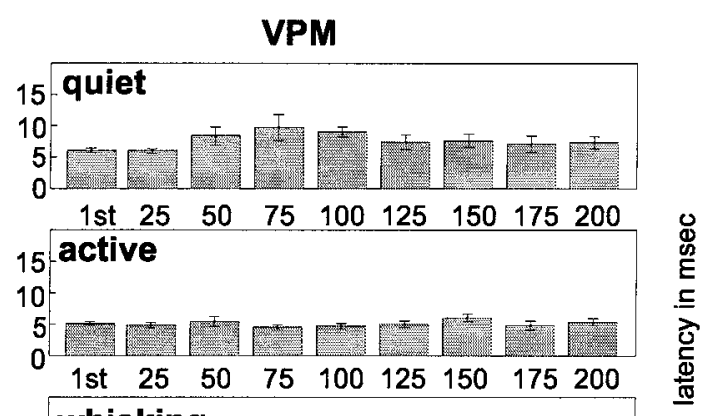

15 -whisking

$10-$

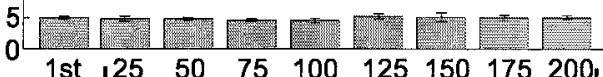

stimulus

time to 2 nd stimulus in $\mathrm{ms}$

C
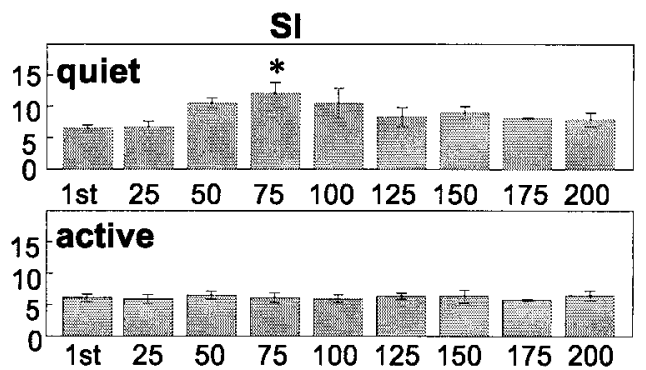

15 whisking

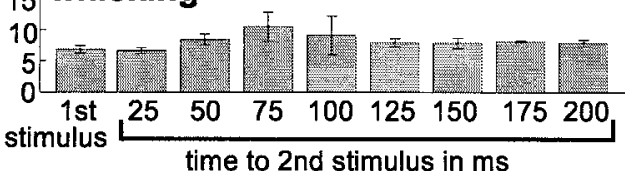

Figure 8. Response latencies in VPM and SI across behavioral states. $a$, Latencies for single stimuli. $b, c$, Latencies for pairs of stimuli. Labeling conventions are described in Figure 5. These latency values were obtained from single-unit and multiunit data combined. ANOVA results: $a$, VPM, $F=35.86, p=0.0003$; SI, $F=18.33, p=0.002 ; b$, quiet, $F=1.55, p=$ 0.19 ; active, $F=0.86, p=0.57$; whisking, $F=5.79, p=0.011$ (no values found significantly different from the first stimulus value); $c$, quiet, $F=$ 3.62, $p=0.013$; active, $F=0.18, p=0.99$; whisking, $F=0.93, p=0.51$. Please see text for $p$ values from post hoc tests (Tukey's HSD).

The magnitude and time course of response suppression to the second stimulus in a pair varied according to behavioral state. Although this suppression effect was significant and long-lasting 
for both VPM and SI during quiet immobility, this was not the case during the active or whisking states. During the active behavioral state, responses to the second stimulus were significantly reduced in VPM and SI only at the $25 \mathrm{msec}$ interstimulus interval and only for the integrated response measure. During the whisking state, sensory responses to the second stimulus were not significantly reduced at any of the interstimulus intervals tested, although there was a nonsignificant trend toward lower values at the $25 \mathrm{msec}$ interstimulus interval (Figs. 5, 6). These results indicated that the suppression of the response to the second of two paired stimuli was substantially smaller and occurred in a shorter interstimulus interval window during active and whisking behaviors compared with quiet immobility.

Because many of the studies of this response suppression effect have been performed in anesthetized animals (Hellweg et al., 1977; Castro-Alamancos and Connors, 1996b), we compared our results in the awake behavioral states described above with results seen in animals under barbiturate anesthesia (Fig. 7). Levels of neuronal-firing activity in the anesthetized preparation before stimulus presentation were much lower than that in the awake animals (compare Figs. 4, 7a). In both VPM and SI, there were reductions in the firing rate after a single stimulus, although they were less robust than the decreases in neuronal activity levels seen in the awake animal. Long-latency excitatory responses started at $\sim 15-35 \mathrm{msec}$ in VPM and SI and lasted up to $70 \mathrm{msec}$ in VPM and $25 \mathrm{msec}$ in SI. In contrast to the extremely longlatency excitatory components observed during the awake quiet state (peaking at $\sim 120 \mathrm{msec}$ after stimulus), no excitatory components were observed in the thalamocortical loop at these poststimulus times under pentobarbital anesthesia [although they have been described under light anesthesia at longer latencies (Armstrong-James and George, 1988)].

Under pentobarbital anesthesia, the time course of the decreases in neural activity in both VPM and SI after the presentation of a stimulus was concurrent with the patterns of response suppression of the second of paired stimuli in these areas. Figure $7 b$ illustrates that in VPM, under pentobarbital anesthesia, a statistically significant reduction in the magnitude of sensory responses started at an interstimulus interval of $50 \mathrm{msec}$ and lasted until $125 \mathrm{msec}$ (maximum magnitude, $p<0.03$; integrated response, $p<0.02$ ). This suppression effect in VPM was both much less robust than and occurred later than that observed in the awake, quiet behavioral state. Unlike all of the awake states observed, there was no reduction in response magnitude at the 25 msec interstimulus interval, although this may be attributable to the fact that VPM neurons exhibit long-latency excitatory responses lasting until at least $25 \mathrm{msec}$ during pentobarbital anesthesia. In contrast, in the SI cortex, response suppression for the second stimulus was much more robust and occurred earlier than in the VPM thalamus under barbiturate anesthesia (Fig. 7c). This cortical suppression was statistically significant for interstimulus intervals ranging from 25 to $125 \mathrm{msec}$ (maximum magnitude, $p<$ 0.04 ; integrated response, $p<0.02$ ). These results indicate that in the anesthetized animal, VPM showed much less suppression than in the awake quiet state, whereas SI showed more response suppression and that suppression was maintained longer than in the awake quiet state. Therefore, it seems that barbiturate anesthesia substantially reduces the mechanism for response suppression in VPM thalamus but increases its duration and intensity in SI cortex, compared with that in the unanesthetized quiet state.

\section{Response latencies}

Behavioral state also influenced the latency of the maximum response magnitude in the rat thalamocortical loop (Fig. 8). For VPM neurons, the average latencies to the maximum shortlatency $(2-20 \mathrm{msec})$ excitatory response were: quiet, $6.0 \pm 0.3$ msec; active, $5.1 \pm 0.3 \mathrm{msec}$; whisking, $5.0 \pm 0.2 \mathrm{msec}$; and whisker twitching, $8.9 \pm 0.5 \mathrm{msec}$. In SI the average latencies for individually presented stimuli were: quiet, $6.5 \pm 0.6 \mathrm{msec}$; active, $6.1 \pm 0.6 \mathrm{msec}$; whisking, $6.8 \pm 0.6 \mathrm{msec}$; and whisker twitching, $9.6 \pm 0.3 \mathrm{msec}$. In both VPM and SI, the response latency in the whisker-twitching state was significantly larger than that in the other three states (see Fig. 8a). Further analysis revealed that for VPM neurons, there was an increase in the average latency for the second stimulus response (in a paired stimulus presentation) during the quiet state, although this increase did not reach statistical significance. In contrast, no increase was observed during the active or whisking states. A similar pattern was observed in SI cortex, and the increase during the quiet state was significant at the $75 \mathrm{msec}$ interstimulus interval $(p<0.04)$.

\section{DISCUSSION}

This study demonstrated that somatosensory responses in both the VPM thalamus and the SI cortex vary substantially according to the behavioral state during which an animal receives a tactile stimulus. First, we showed that neuronal responses in the whisker representations of VPM and SI to single stimuli were significantly reduced during periods of active vibrissal movements (whisking and whisker-twitching behaviors) compared with periods when no vibrissal movements were observed (quiet and active behavioral states). Second, the ability of thalamic and cortical neurons to respond to repeated tactile stimuli was greatest during the whisking and active states but was substantially reduced during the quiet state for interstimulus intervals ranging from 25 to 75 msec.

These results support the hypothesis that the characteristics of tactile responses in the rat somatosensory system can be dynamically altered depending on the behavioral state during which tactile stimuli are presented. On the basis of these data, we propose that processing of tactile stimuli by the somatosensory system is modulated according to the animal's behavior to maximize the detection of different types of tactile information. Specifically, in the absence of whisker movements (quiet immobility), both VPM and SI neurons seem to be highly responsive to punctate tactile stimuli but not to sequences of stimuli. In contrast, during the whisking state, when active exploratory whisker movements are used by the rat to gather tactile information, the temporal fidelity of sensory responses to rapidly presented stimuli is enhanced.

\section{Behavioral modulation of short-latency excitatory responses to individual tactile stimuli}

We observed that both cortical and thalamic short-latency excitatory sensory responses $(2-20 \mathrm{msec})$ to single tactile stimuli were largest during the quiet and active states, when no whisker movements were observed. In contrast, the sizes of these responses were reduced overall when the animal was engaged in exploratory whisker movements or whisker-twitching behavior. This showed that response magnitude was decreased when there was motor activity that was specific to the vibrissae (i.e., during whisking and whisker twitching) but not when there was motor activity of other areas, such as the limbs, head, or trunk (i.e., during the active behavioral state). These results corroborate other studies of 
motor-induced sensory gating in rats (Chapin and Woodward, 1981, 1982a,b), cats (Ghez and Lenzi, 1971; Coulter, 1974), and monkeys (Nelson, 1984, 1987; Nelson et al., 1991) and further support the demonstration that such gating is specific to the area of the body that is engaged in motor activity (Ghez and Lenzi, 1971; Coulter, 1974; Nelson, 1984, 1987; Nelson et al., 1991). In our analyses, similar results were obtained for both single-unit and multiunit data. In the thalamic recordings, this can be accounted for by the fact that VPM contains a homogeneous population of neurons (thalamocortical-projecting neurons).

One could postulate that the increases in baseline activity levels during the whisking state and, in some cases, the whiskertwitching state could themselves be responsible for the decrease in response magnitudes because of the phenomenon of occlusion. That is, in these states the neurons could be firing too rapidly to respond robustly when a stimulus is presented. We feel this is not a likely scenario here because, despite the increased firing rates in these cases, the neurons were not firing at high enough rates to prevent additional action potentials from occurring. In fact, the background firing rates in the whisking and whisker-twitching states did not approach the firing rates observed during responses to stimuli presented in the quiet state (see Fig. 2). Therefore, it is highly unlikely that the higher background firing rates observed during the whisking and whisker-twitching states were saturating a neuron's ability to fire when a stimulus was presented.

There are several reasons to believe that the mechanism by which tactile responses are reduced during motor activity may be, at least in part, centrally modulated. First, previous studies have shown that response amplitude is reduced more the further removed cells are from the periphery (Chapman et al., 1988; Shin and Chapin, 1989; Shin et al., 1993, 1994), and this pattern was evident in our study during the whisker-twitching behavior. This indicates that this response reduction effect cannot simply be a feedforward effect from the periphery but is likely to involve a degree of central modulation. Second, it has been shown that this type of sensory gating can occur up to $170 \mathrm{msec}$ before the onset of a given movement (Ghez and Lenzi, 1971; Nelson, 1984, 1987), suggesting that the modulation is not caused by the movement itself but is caused by some influence that is engaged before actual muscle activation, such as activity in motor cortex (but see Chapman et al., 1988). In support of this view, Nelson (1984, 1987) noted that reductions in SI responses before motor activity in primates occur in the areas of SI that receive corticocortical projections from the primary motor cortex $(3 a, 1,2)$ but are not observed in area $3 \mathrm{~b}$, which does not receive these direct projections. Because the rat SI cortex has reciprocal connections with primary motor cortex, the substantial sensory gating observed in rat SI in this and other studies (Chapin and Woodward, 1981, $1982 \mathrm{a}, \mathrm{b})$ could result from corticocortical interactions as proposed by Nelson. In support of this view, we observed that the strongest gating effect in SI occurred during periods of whiskertwitching movements, which are known to be dependent on primary motor cortex because they are abolished after ablation of the rat frontal cortex (Semba and Komisaruk, 1984).

It is possible, however, that the trigeminal nucleus may also play a role in the gating effects we observed here. For example, Shin and Chapin (1989) described small amounts of response suppression (overall mean suppression, -7.6\%; maximum, $-27.8 \%$ ) in the dorsal column nuclei of rats in response to MI stimulation. Such modulation in the brainstem could then be passed on to the thalamus and cortex in a feedforward manner. Thus, the results we have described do not necessarily mean that the effects originate in the thalamocortical loop but merely that the neurons in these areas are affected by movement via some as yet unknown mechanism.

\section{Behavioral modulation of postexcitatory firing suppression and long-latency responses}

Different behavioral states also modulated both the amount and duration of postexcitatory firing suppression and long-latency excitatory components of VPM and SI neuronal responses. When compared with the quiet state, motor activity of any sort (i.e., during the active, whisking, and whisker-twitching states) tended to reduce the amount and duration of the period of suppressed firing that followed the short-latency excitatory component of thalamic and cortical neuronal responses. This effect was most pronounced in SI cortex where the period of postexcitatory firing suppression almost disappeared during the active, whisking, and whisker-twitching states. Moreover, long-latency excitatory components of VPM and SI neurons, observed during quiet immobility, were virtually nonexistent in the other three states. These modulations in firing rate may account for the patterns of thalamic and cortical responses observed when pairs of stimuli were presented at varying intervals.

Postexcitatory firing suppression has been characterized in multiple structures of the somatosensory system of several species, including the cat somatosensory system (Mountcastle et al., 1957; Hellweg et al., 1977; Morin and Steriade, 1981; Steriade and Morin, 1981), the monkey primary somatosensory cortex (Gardner and Costanzo, 1980), and the rat barrel cortex (Simons, 1985; Simons and Carvell, 1989). In the barbiturate-anesthetized cat (Hellweg et al., 1977), reductions in firing rates lasting 50-100 msec were observed in cells of the somatosensory cortex after deflection of the vibrissae. Intracellular recordings showed that this effect was paralleled by IPSPs that followed stimulus presentations. Likewise, Simons and Carvell (1989) showed that responses in both VPM and SI to vibrissal stimulation in sedated, paralyzed rats were followed by inhibition of firing. They demonstrated that the population profiles of neural firing in these areas paralleled changes in membrane potentials recorded intracellularly after vibrissal deflection. In addition, their study showed that this pattern corresponded to the time period of cross-whisker inhibition. These results support the finding that inhibitory influences are present immediately after the shortlatency excitatory component of somatosensory neuronal responses. Because the rat VPM contains a homogeneous population of glutamatergic thalamocortical-projecting neurons, its only source of GABAergic afferents is the neurons located in the reticular nucleus of the thalamus (RT). RT neurons receive feedforward glutamatergic collaterals from VPM axons and feedback projections from corticothalamic neurons located in different cortical areas of the rat somatosensory system. It is likely that descending afferents from the cortex could alter the amount of inhibition in VPM by modulating the firing of these RT neurons. However, this postexcitatory firing suppression effect could also be a feedforward effect from inhibition of firing that could occur in the trigeminal nucleus after a stimulus.

Further evidence that the period of reduced firing after a stimulus can be modified by different brain states comes from a study by Steriade and Morin (1981) of the augmenting response in cat ventrobasal thalamus (VB) and SI. They demonstrated that firing after the response triggered by stimulation of either $\mathrm{VB}$ or the white matter below SI in VB-lesioned animals was reduced when the stimulus was closely preceded by electrical stimulation 
of the brainstem reticular formation. As in our study, the shortening of the period of reduced firing after a stimulus was also accompanied by an increase in the overall spontaneous firing rate. On the basis of this evidence, it is possible that the reduction in suppressed firing we observed after a stimulus in the whisking and active states could be influenced by reticular formation activity or by other cholinergic projections to the thalamocortical loop.

Analogous behavioral modulation of responses has been observed in other studies of the augmenting response. CastroAlamancos and Connors (1996a) demonstrated that when an animal was quiescent, stimulation in the forelimb representation in the ventrolateral (motor) thalamus would increase the response to a stimulus presented $100 \mathrm{msec}$ later. However, this augmenting response disappeared when the animal was engaged in grasping behaviors involving the paw. Despite the fact that the augmenting response involves an increase in activity, whereas the effect presented in this report involves response suppression, there is a similarity between the reduction of the augmented response during motor activity in Castro-Alamancos and Connors's studies and the reduction of tactile response suppression seen in this paper. It seems that during motor activity both types of cortical responses (second stimulus suppression and augmenting responses) may be suppressed by a "central gating mechanism."

\section{Behavioral modulation of responses to pairs of stimuli}

When stimuli were delivered in pairs at varying interstimulus intervals, the magnitude of the neuronal response to the second stimulus in a pair varied according to behavioral state. During the quiet behavioral state, the response to the first stimulus could partially or entirely suppress the response to the second stimulus if the interstimulus interval was in the $25-75 \mathrm{msec}$ range. These results are very similar in time course to those obtained by Laskin and Spencer (1979) in the somatosensory cortex of unanesthetized, paralyzed cats in response to pairs of air puffs applied to the forepaw at varying interstimulus intervals. In contrast, during the whisking and active behavioral states, the period of time during which this response suppression could be observed was reduced, occurring only when the interstimulus interval was 25 msec. Thus, the somatosensory system's ability to respond to rapidly repeated stimuli was enhanced during the whisking and active states, a finding that is consistent with the fact that during exploratory behavior, the somatosensory system may need to integrate tactile information that results from multiple rapidly presented whisker contacts.

Interestingly, the differing time courses of this response suppression effect during the four behavioral states studied here were very similar to the time courses of postexcitatory firing suppression observed during those states. The period in the quiet state during which there was a substantial reduction in the response to the second stimulus of a pair mirrored the period during which neuronal activity was reduced below the baseline firing rate (the postexcitatory period of firing suppression) in both VPM and SI. In contrast, during the whisking and active states, the period of postexcitatory firing suppression was substantially reduced in VPM and virtually nonexistent in SI. Thus, the shortening of the period during which a significant attenuation of the second stimulus response was observed was correlated with either the reduction (VPM) or elimination (SI) of the postexcitatory firing suppression period during the active and whisking states.

\section{Optimization of tactile processing under different behavioral conditions}

The evidence presented above demonstrates that thalamic and cortical somatosensory response characteristics are not static but instead can be modulated during different types of motor activity. Motor activity affects not only the responses to individually presented stimuli but also the manner in which neurons respond to repeated stimuli. These factors are important for understanding how the somatosensory system processes tactile information and suggest that neuronal response properties as well as the spatiotemporal characteristics of VPM and SI receptive fields are not labile entities (Nicolelis and Chapin, 1994; Ghazanfar and Nicolelis, 1999) but can be modulated according to an animal's behavioral state. One possible interpretation of these results is that somatosensory response properties are modulated to optimize the type of stimulus characteristics that are to be detected under different behavioral conditions. In particular, we speculate that during the quiet behavioral state, a period in which there is no active use of the whiskers for tactile exploration or discrimination, the vibrissal system is highly sensitive to any punctate stimulus to the whiskers. However, in this state, sensitivity to repeated stimuli, such as would occur to an individual whisker as it brushes across a textured surface, is reduced. In contrast, in behavioral states in which the whiskers are used in active exploration, as during the whisking behavioral state, when sequences of tactile stimuli are expected and may be used for discrimination of object features such as texture and shape, fidelity of responses to rapidly presented stimuli is enhanced, so more nuances of a tactile stimulus can be detected. This could allow the whiskers to acquire a more complete and continuous representation of the environment being investigated. However, sensitivity to individual stimuli under these conditions seems to be reduced. Both stimulus detection and the ability to follow rapidly repeated stimuli appear to be facilitated during the active state when animals are not explicitly using their whiskers but are in an attentive, alert state. Although the behavioral function of the whisker-twitching state is unknown, the results presented here suggest that although normal sensory responses can be clearly recognized across the thalamocortical loop during this behavior, significant gating of these responses does occur. This trade-off between sensitivity and fidelity of repeated stimuli may allow the nervous system to optimize detection of different types of stimuli that can be encountered under different behavioral conditions.

\section{REFERENCES}

Armstrong-James M, George MJ (1988) Influence of anesthesia on spontaneous activity and receptive field size of single units in rat Sm1 neocortex. Exp Neurol 99:369-387.

Carvell GE, Simons DJ (1990) Biometric analyses of vibrissal tactile discrimination in the rat. J Neurosci 10:2638-2648.

Castro-Alamancos MA, Connors BW (1996a) Short-term plasticity of a thalamocortical pathway dynamically modulated by behavioral state. Science 272:274-277.

Castro-Alamancos MA, Connors BW (1996b) Spatiotemporal properties of short-term plasticity sensorimotor thalamocortical pathways of the rat. J Neurosci 16:2767-2779.

Chapin JK, Woodward DJ (1981) Modulation of sensory responsiveness of single somatosensory cortical cells during movement and arousal behaviors. Exp Neurol 72:164-178.

Chapin JK, Woodward DJ (1982a) Somatic sensory transmission to the cortex during movement: gating of single cell responses to touch. Exp Neurol 78:654-669.

Chapin JK, Woodward DJ (1982b) Somatic sensory transmission to the cortex during movement: phasic modulation over the locomotor step cycle. Exp Neurol 78:670-684. 
Chapman CE, Jiang W, Lamarre Y (1988) Modulation of lemniscal input during conditioned arm movements in the monkey. Exp Brain Res 72:316-334.

Coulter JD (1974) Sensory transmission through lemniscal pathway during voluntary movement in the cat. J Neurophysiol 37:831-845.

Fanselow EE, Rosen MJ, Nicolelis MAL (1997) Behavioral modulation of tactile responses in the rat thalamocortical loop. New Orleans: Society for Neuroscience.

Gardner EP, Costanzo RM (1980) Temporal integration of multiplepoint stimuli in primary somatosensory cortical receptive fields of alert monkeys. J Neurophysiol 43:444-468.

Ghazanfar AA, Nicolelis MA (1997) Nonlinear processing of tactile information in the thalamocortical loop. J Neurophysiol 78:506-510.

Ghazanfar AA, Nicolelis MA (1999) Spatiotemporal properties of layer $\mathrm{V}$ neurons of the rat primary somatosensory cortex. Cereb Cortex 9:348-361.

Ghez C, Lenzi GL (1971) Modulation of sensory transmission in cat lemniscal system during voluntary movement. Pflügers Arch 323:273-278.

Grill WM, Mortimer JT (1998) Stability of the input-output properties of chronically implanted multiple contact nerve cuff stimulating electrodes. IEEE Trans Rehabil Eng 6:364-373.

Hellweg FC, Schultz W, Creutzfeldt OD (1977) Extracellular and intracellular recordings from cat's cortical whisker projection area: thalamocortical response transformation. J Neurophysiol 40:463-479.

Laskin SE, Spencer WA (1979) Cutaneous masking. II. Geometry of excitatory and inhibitory receptive fields of single units in somatosensory cortex of the cat. J Neurophysiol 42:1061-1082.

Lebedev MA, Denton JM, Nelson RJ (1994) Vibration-entrained and premovement activity in monkey primary somatosensory cortex. J Neurophysiol 72:1654-1673.

Morin D, Steriade M (1981) Development from primary to augmenting responses in the somatosensory system. Brain Res 205:49-66.

Mountcastle VB, Davies PW, Berman AL (1957) Response properties of neurons of cat's somatic sensory cortex to peripheral stimuli. J Neurophysiol 20:374-407.

Nelson RJ (1984) Responsiveness of monkey primary somatosensory cortical neurons to peripheral stimulation depends on "motor-set." Brain Res 304:143-148.

Nelson RJ (1987) Activity of monkey primary somatosensory cortical neurons changes prior to active movement. Brain Res 406:402-407.

Nelson RJ, Li B, Douglas VD (1991) Sensory response enhancement and suppression of monkey primary somatosensory cortical neurons. Brain Res Bull 27:751-757.
Nicolelis MA, Chapin JK (1994) Spatiotemporal structure of somatosensory responses of many-neuron ensembles in the rat ventral posterior medial nucleus of the thalamus. J Neurosci 14:3511-3532.

Nicolelis MA, Baccala LA, Lin RC, Chapin JK (1995) Sensorimotor encoding by synchronous neural ensemble activity at multiple levels of the somatosensory system. Science 268:1353-1358.

Nicolelis MA, Ghazanfar AA, Faggin BM, Votaw S, Oliveira LM (1997) Reconstructing the engram: simultaneous, multisite, many single neuron recordings. Neuron 18:529-537.

O'Keefe J, Gaffan D (1971) Response properties of units in the dorsal column nuclei of the freely moving rat: changes as a function of behaviour. Brain Res 31:374-375.

Paxinos G, Watson C (1986) The rat brain. San Diego: Academic.

Semba K, Komisaruk BR (1984) Neural substrates of two different rhythmical vibrissal movements in the rat. Neuroscience 12:761-774.

Shin HC, Chapin JK (1989) Mapping the effects of motor cortex stimulation on single neurons in the dorsal column nuclei in the rat: direct responses and afferent modulation. Brain Res Bull 22:245-252.

Shin HC, Chapin JK (1990a) Modulation of afferent transmission to single neurons in the ventroposterior thalamus during movement in rats. Neurosci Lett 108:116-120.

Shin HC, Chapin JK (1990b) Movement induced modulation of afferent transmission to single neurons in the ventroposterior thalamus and somatosensory cortex in rat. Exp Brain Res 81:515-522.

Shin HC, Park HJ, Chapin JK (1993) Differential phasic modulation of short and long latency afferent sensory transmission to single neurons in the ventroposterolateral thalamus in behaving rats. Neurosci Res $17: 117-125$.

Shin HC, Park HJ, Chapin JK (1994) Differential phasic modulation of short and long latency afferent sensory transmission to single neurons in the primary somatosensory cortex in behaving rats. Neurosci Res 19:419-425.

Simons DJ (1985) Temporal and spatial integration in the rat SI vibrissa cortex. J Neurophysiol 54:615-635.

Simons DJ, Carvell GE (1989) Thalamocortical response transformation in the rat vibrissa/barrel system. J Neurophysiol 61:311-330.

Steriade M, Morin D (1981) Reticular influences on primary and augmenting responses in the somatosensory cortex. Brain Res 205:67-80.

Weliky M, Katz LC (1997) Disruption of orientation tuning in visual cortex by artificially correlated neuronal activity [see comments]. Nature 386:680-685.

Welker WI (1964) Analysis of sniffing of the albino rat. Behavior 22: 223-244. 